\title{
Graphene and Carbon Nanotubes in Space
}

\author{
Xiuhui Chen ${ }^{1}$, Zichun Xiao ${ }^{2}$, Aigen $\mathbf{L i}^{3}$, and Jianxin Zhong ${ }^{1}$ \\ ${ }^{1}$ Xiangtan University, China; ${ }^{2}$ Ravenscroft School, Raleigh, NC 27615, USA; ${ }^{3}$ University of \\ Missouri, USA; email: lia@missouri.edu
}

As the fourth most abundant element in the universe, carbon plays an important role in the physical and chemical evolution of the interstellar medium (ISM). Due to its unique property to form three different types of chemical bonds through $\mathrm{sp}^{1}, \mathrm{sp}^{2}$, and $\mathrm{sp}^{3}$ hybridizations, carbon can be stabilized in various allotropes, including amorphous carbon, graphite, diamond, polycyclic aromatic hydrocarbon (PAH), fullerenes, graphene, and carbon nanotubes (CNTs).

Many allotropes of carbon are known to be present in the ISM (Henning \& Salama 1998). Presolar graphite grains and nanodiamonds have been identified in primitive meteorites based on their isotopically anomalous composition (see Nittler 2018). While hydrogenated amorphous carbon grains reveal their presence in the diffuse ISM through the ubiquitous $3.4 \mu \mathrm{m}$ aliphatic $\mathrm{C}-\mathrm{H}$ absorption feature (Pendleton \& Allamandola 2002), the aromatic $\mathrm{C}-\mathrm{H}$ and $\mathrm{C}-\mathrm{C}$ emission features at 3.3, 6.2, 7.7, 8.6 and $11.3 \mu \mathrm{m}$ infer the widespread presence of PAHs in a wide variety of interstellar regions (see Hudgins \& Allamandola 2005). The detections of interstellar $\mathrm{C}_{60}$ and $\mathrm{C}_{70}$ (Cami et al. 2010, Sellgren et al. 2010) and their cations (Berné et al. 2013, Strelnikov et al. 2015) have also been reported based on their characteristic infrared (IR) emission features.

Graphene was first experimentally synthesized in 2004 by A.K. Geim and K.S. Novoselov for which they received the 2010 Nobel Prize in physics. More recently, García-Hernández et al. $(2011,2012)$ reported for the first time the presence of unusual IR emission features at $\sim 6.6,9.8$, and $20 \mu \mathrm{m}$ in several planetary nebulae, both in the Milky Way and the Magellanic Clouds, which are coincident with the strongest transitions of planar $\mathrm{C}_{24}$, a piece of graphene. In principle, graphene could be present in the ISM as it could be formed from the photochemical processing of PAHs, which are abundant in the ISM, through a complete loss of their H atoms (e.g., see Berné \& Tielens 2012). On the other hand, as illustrated in Figure $1 \mathrm{l}$, both quantum-chemical computations and laboratory experiments have shown that the exciton-dominated $\pi-\pi^{*}$ electronic transitions in graphene cause a strong absorption band near $2755 \AA$ or $4.5 \mathrm{eV}$ (Yang et al. 2009, Nelson et al. 2010 ) which is not seen in the ISM. This allows us to place an upper limit of $\sim 5 \mathrm{ppm}$ of $\mathrm{C} / \mathrm{H}$ on the abundance of graphene in the diffuse ISM (see Chen et al. 2017). Moreover, the nondetection of the $6.6,9.8$, and $20 \mu \mathrm{m}$ emission features of graphene $\mathrm{C}_{24}$ in the observed IR emission spectra of the diffuse ISM is also consistent with an upper limit of $\sim 5 \mathrm{ppm}$ of $\mathrm{C} / \mathrm{H}$ in the $\mathrm{C}_{24}$ graphene sheet (see Chen et al. 2017).

CNTs can be envisioned as a layer of graphene sheet rolled up into a cylinder. They are novel 1D materials made of an $\mathrm{sp}^{2}$-bonded wall one atom thick. Strong confinement $(\sim 1 \mathrm{~nm})$ of charge carriers results in their unique optical properties being dominated by strongly bound excitons as revealed by the sharp optical absorption features. It would be interesting to explore whether CNTs could be responsible for some of the mysterious diffuse interstellar bands (e.g., see Zhou et al. 2006).

As illustrated in Figure $1 \mathrm{~b}$, CNTs also exhibit a broad and intense absorption feature at $\sim 4.5 \mathrm{eV}$ that is typically attributed to a $\pi$-plasmon excitation (e.g., see Kataura et al. 1999), although the exact position of this feature appears to vary with nanotube diameter 

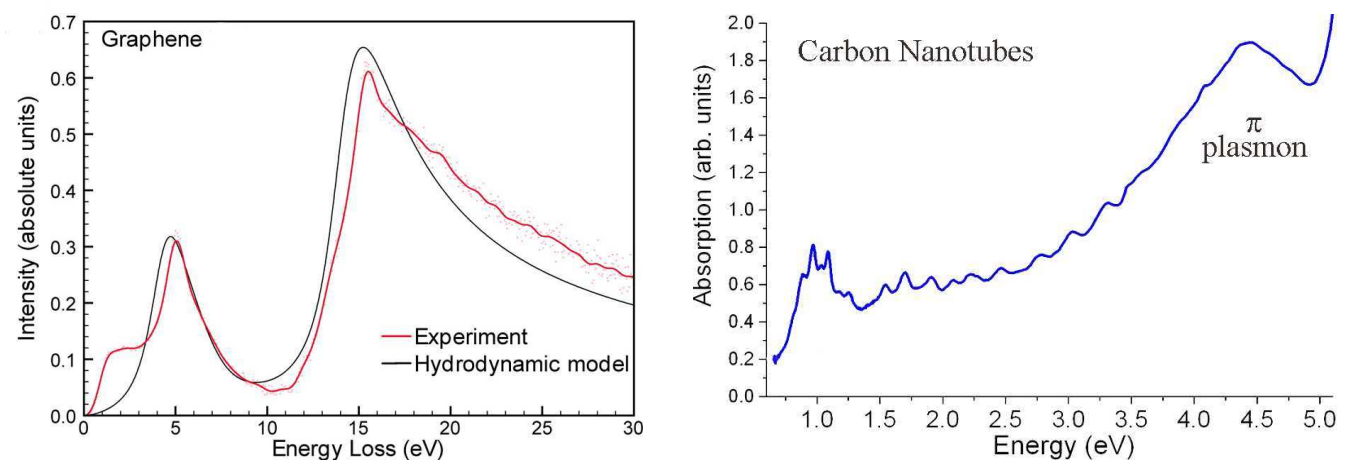

Figure 1. Left (a): The energy-loss function (ELF) of graphene, obtained experimentally and calculated using a two-dimensional hydrodynamic model by Nelson et al. (2014), is dominated by two peaks at $\sim 4.5$ and $\sim 15 \mathrm{eV}$, known as $\pi$ and $\pi+\sigma$ plasmons, respectively. Right (b): The optical absorption spectrum from dispersed single-wall CNTs measured by Kataura et al. (1999) exhibits a number of electronic transitons, with the $\pi$ plasmon also peaking at $\sim 4.5 \mathrm{eV}$ being the most prominent. The nondetection in the Galactic interstellar extinction curve of the $\sim 4.5 \mathrm{eV}$ absorption peak of graphene and CNTs would allow one to place an upper limit on the amounts of graphene and CNTs in the ISM.

(see Rance et al. 2010). Similar to graphene, the absence of the $\sim 4.5 \mathrm{eV}$ absorption in the ISM would allow us to place an upper limit on the abundance of interstellar CNTs.

Like graphene, CNTs would emit in the IR through their $\mathrm{C}-\mathrm{C}$ vibrational modes. CNTs are actually more IR-active than graphene due to their cylindrical boundary condition. Many vibrational modes in the $\sim 680-1730 \mathrm{~cm}^{-1}$ range have been experimentally detected for CNTs (e.g., see Kim et al. 2005). JWST's unique high sensitivity and high resolution IR capabilities will open up an IR window unexplored by Spitzer and unmatched by ISO observations and thus will allow us to explore the possible presence of graphene and CNTs in the ISM in greater detail.

Acknowledgements: We are supported in part by NSF AST-1816411 and an AAS international travel grant.

\section{References}

Berné, O., \& Tielens, A.G.G.M. 2012, PNAS, 109, 401

Berné, O., Mulas, G., \& Joblin, C. 2013, A\&A, 550, L4

Cami, J., Bernard-Salas, J., Peeters, E., \& Malek, S. E. 2010, Science, 329, 1180

Chen, X.H., Li, A., \& Zhang, K. 2017, ApJ, 850, 104

García-Hernández, D. A., Kameswara Rao, N., \& Lambert, D. L. 2011, ApJ, 729, 126

García-Hernández, D. A., Villaver, E., García-Lario, P., et al. 2012, ApJ, 760, 107

Henning, Th., \& Salama, F. 1998, Science, 282, 2204

Hudgins, D. M., \& Allamandola, L. J. 2005, IAU Symp. 231, 443

Kataura, H., Kumazawa, Y., Maniwa, Y., et al. 1999, Synth. Metals, 103, 2555

Kim, U.J., Liu, X.M., Furtado, C.A., et al. 2005, Phys. Rev. Lett., 95, 157402

Nelson, F. J., Kamineni, V. K., Zhang, T., et al. 2010, Appl. Phys. Lett., 97, 253110

Nelson, F. J., Idrobo, J.-C., Fite, J.D., et al. 2014, Nano Lett., 14, 3827

Nittler, L.R. 2018, Geochim. Cosmochim. Acta, 221, 1

Pendleton, Y. J., \& Allamandola, L. J. 2002, ApJS, 138, 75

Rance, G.A., Marsh, D.H., Nicholas, R.J., et al. 2010, Chem. Phys. Lett., 493, 19

Sellgren, K., Werner, M. W., Ingalls, J. G., et al. 2010, ApJL, 722, L54

Strelnikov, D., Kern, B., \& Kappes, M. M. 2015, A\& A, 584, A55

Yang, L., Deslippe, J., Park, C.-H., et al. 2009, Phys. Rev. Lett., 103, 186802

Zhou, Z., Sfeir, M. Y., Zhang, L., et al. 2006, ApJL, 638, L105 